\title{
Quantitative evaluation of performance of three-dimensional printed lenses
}

\author{
John Gawedzinski, ${ }^{a}$ Michal E. Pawlowski, ${ }^{a}$ and Tomasz S. Tkaczyk ${ }^{a, b, \star}$ \\ ${ }^{a}$ Rice University, Department of Bioengineering, Houston, Texas, United States \\ ${ }^{\mathrm{b}}$ Rice University, Electrical and Computer Engineering, Houston, Texas, United States
}

\begin{abstract}
We present an analysis of the shape, surface quality, and imaging capabilities of custom three-dimensional (3-D) printed lenses. 3-D printing technology enables lens prototypes to be fabricated without restrictions on surface geometry. Thus, spherical, aspherical, and rotationally nonsymmetric lenses can be manufactured in an integrated production process. This technique serves as a noteworthy alternative to multistage, labor-intensive, abrasive processes, such as grinding, polishing, and diamond turning. Here, we evaluate the quality of lenses fabricated by Luxexcel using patented Printoptical ${ }^{\odot}$ technology that is based on an inkjet printing technique by comparing them to lenses made with traditional glass processing technologies (grinding, polishing, etc.). The surface geometry and roughness of the lenses were evaluated using white-light and Fizeau interferometers. We have compared peak-to-valley wavefront deviation, root mean square (RMS) wavefront error, radii of curvature, and the arithmetic roughness average ( $\mathrm{Ra}$ ) profile of plastic and glass lenses. In addition, the imaging performance of selected pairs of lenses was tested using 1951 USAF resolution target. The results indicate performance of 3-D printed optics that could be manufactured with surface roughness comparable to that of injection molded lenses $(\mathrm{Ra}<20 \mathrm{~nm})$. The RMS wavefront error of 3-D printed prototypes was at a minimum 18.8 times larger than equivalent glass prototypes for a lens with a $12.7 \mathrm{~mm}$ clear aperture, but, when measured within $63 \%$ of its clear aperture, the 3-D printed components' RMS wavefront error was comparable to glass lenses. ๑ 2017 Society of Photo-Optical Instrumentation Engineers (SPIE) [DOI: 10.1117/1.OE.56.8.084110]
\end{abstract}

Keywords: three-dimensional printing of optical components; lens fabrications; lens characterization.

Paper 170509 received Apr. 5, 2017; accepted for publication Jul. 27, 2017; published online Aug. 30, 2017.

\section{Introduction}

Optical elements can be manufactured through a variety of techniques. Grinding and polishing are well-established methods used to manufacture precise optical elements. Traditionally, lenses for the visible part of the optical spectrum are made from glass, and materials such as crystals and metals are used to build systems in the infrared and ultraviolet part of the electromagnetic spectrum. ${ }^{1-3}$ Today, mass-produced optical components are often made using polymers. Polymer-specific processes, such as injection ${ }^{4}$ or compression ${ }^{5}$ molding can be tuned for high-volume manufacturing. While tooling and tuning of the production process for those techniques is both labor-intensive and costly, efficiency and high rate of production results in a low overall cost of mass-produced components. Currently, these stateof-the-art lens fabrication techniques permit the production of parts with surface quality on the nanometer scale (roughness, form), but each method faces challenges when trying to prototype optics of complex geometries. Traditional grinding and polishing techniques are not optimal for production of non-spherical components, ${ }^{1}$ and existing techniques for creating aspherical components are laborious and costly. ${ }^{6-8}$ Recent advances in numerically controlled magnetorheological machining has enabled the production of high-quality free-form optical components, though these processes still require lengthy part-processing and experience problems with production scalability. ${ }^{9-11}$ For molding techniques,

*Address all correspondence to: Tomasz S. Tkaczyk, E-mail: ttkaczyk@rice .edu the shape of the lens is defined by mold geometry, which makes it possible to mass-produce spherical, aspherical, and free-form optics without additional cost at high volumes. ${ }^{2,10}$ However, molding techniques are limited by the availability or production costs of the molds themselves, which can often range as high as $\$ 25,000$ and can be impractical for prototyping. ${ }^{2}$ Prototyping using nonferrous materials is best served by diamond-turning technology. ${ }^{12-15}$ Unlike other manufacturing technologies, diamond turning allows for direct prototyping of refractive, reflective, and diffractive elements without the need for subsequent postprocessing steps. The main disadvantage of diamond turning technology, especially for wavelengths below the infrared region, is scattering of manufactured components due to the high-frequency residual imprint of a diamond tool.

Three-dimensional (3-D) printing technology introduced at the beginning of $1990^{16}$ is quickly gaining market share for prototyping and short-scale production. ${ }^{17}$ Originally thought of as a tool to manufacture evaluation prototypes for research and development departments and as a tool to simplify mold production, 3-D printing has quickly evolved into a technique used in both industry and academia and appears poised to revolutionize consumer markets in the near future. ${ }^{18-23}$ With recent progress and available materials, it is now possible to print structural, electrically conductive, ${ }^{24}$ and magnetic parts ${ }^{25}$ used in integrated electromechanical systems. ${ }^{26}$ As prototyping of mechanical components progresses rapidly and prototyping of functional

0091-3286/2017/\$25.00 (C) 2017 SPIE 
mechanical components is currently a well-established technology, 3-D printing of optical components has just emerged as another application of this technology. Printing optics is challenging due to the specific requirements of high purity and uniformity of fabricated components that go together with tight geometrical tolerances that are typically on the order of a fraction of the wavelength. Functional optical components, such as aspheric lenses and waveguides, have been successfully printed for the terahertz range. ${ }^{27-30}$ In $\mathrm{THz}$ regime, mechanical precision of most of the 3-D printing machines that is on the order of tens of millimeter is acceptable as those tolerances are smaller or comparable to the wavelength of propagating radiation. However, optics manufactured for the visible range of the electromagnetic spectrum must have geometric tolerances that are on order of nanometers, which is out of range for most current 3-D printing machines. In the visible region, examples of successfully manufactured optical components include light guides, transparent windows, fibers, opto- electro-mechanical sensors, and small lenses. ${ }^{24,26,28}$ Recent attempts to create simple lenses using inkjet-like printing technology resulted in the synthesis of small $(<7 \mathrm{~mm})$ flexible lenses that can be attached to smartphones and potentially used for microscopy applications. ${ }^{31}$ However, the size of lenses manufactured using this technique is limited by surface tension to diameters on the order of millimeters, with little control of surface geometry. Luxexcel has recently developed a manufacturing technique that enables the production of free-form optical components. These components are manufactured using a UV-curable photopolymer with a refractive index of 1.53 that is jetted onto a poly(methyl methacrylate) (PMMA) substrate with a refractive index of $1.49 .{ }^{32}$ Subsequently, the polymer is allowed to set before the fabricated lenses are cured to a solid state using UV light, effectively hardening the photopolymer into a desired shape. This combinational technique of inkjet printing and molding enables prototyping of optical components in the range between 1 and $180 \mathrm{~mm}$ in diameter with arbitrary shapes, ultimately favoring planospherical and aspherical lenses. Manufacturing only requires a specific computer-aided design model. This technique does not require postprocessing and, unlike other optical manufacturing techniques, such as polishing and diamond turning, does not need to be tooled specifically for a manufactured part.

Here, we evaluate current state of Printoptical ${ }^{\circledR}$ Luxexcel technology through comparison of 3-D printed lenses to geometrically equivalent lenses obtained from Edmund Optics and Thorlabs. To perform fair comparison and simplify testing procedures between state-of-the-art glass lenses and 3-D printed lenses, we selected spherical components. Lenses with apertures ranging from 10 to $50 \mathrm{~mm}$ were purchased from Edmund Optics and Thorlabs. Design schematics and parameters details were then sent to Luxexcel, and equivalent 3-D printed lenses were acquired. The Luxexcel polymer properties including index of refraction, transmission, and autofluorescence were measured as a part of evaluation procedures. The refractive index was characterized between 486 and $656 \mathrm{~nm}$ using a refractometer. A spectrophotometer was used to characterize transmission of the material between 310 and $1080 \mathrm{~nm}$, and a fluorimeter with excitation wavelengths ranging from 300 to $750 \mathrm{~nm}$ was used to measure the autofluorescence profile. White- light interferometric data were acquired for both the Luxexcel lenses and the reference lenses to compare surface roughness. Fizeau interferometry was applied to measure the surface figure. Finally, we imaged a 1951 USAF resolution target using a 3-D printed lens and glass substitutes. An optical system comprising a microscope illumination system, a narrowband interference filter, an adjustable aperture stop, and a monochromatic detector was used to evaluate the resolution limit of both lenses working in $1 \times$ magnification.

\section{Methods}

Six glass lenses were purchased from Edmund Optics and Thorlabs to serve as quality references, and their geometries are summarized in Table 1. All lenses were plano-convex except for Edmund Optics lens 63536, which was a biconvex lens. For each of the six reference lenses, three equivalent Luxexcel lenses were purchased and printed. In total, we tested 18 lenses. In addition, a single $8 \mathrm{~mm} \times 15 \mathrm{~mm} \times$ $3 \mathrm{~mm}$ solid rectangular prism was purchased from Luxexcel for material characterization purposes. All investigated components are shown in Fig. 1.

The refractive index of the Luxexcel Opticlear ${ }^{\circledR}$ material was measured on an Atago multiwavelength Abbe DR-M4 refractometer. The refractometer was calibrated with a test piece of a known refractive index $(n=1.6199)$ prior to all measurements. Each measurement was taken at room temperature with a thin layer $(<1 \mathrm{~mm})$ of monobromonaphthalene employed as a contact liquid. The material refractive index was quantified at four discrete wavelengths, using 486, 546, 589, and $656 \mathrm{~nm}$ filters. Measurements were taken three times, and the results were averaged. Transmission of the Luxexcel Printoptical ${ }^{\complement}$ material was characterized using a Varian Cary 50 UV-Vis spectrophotometer. After performing a baseline calibration measurement, transmission of a Luxexcel sample block was measured across a wavelength range of 310 to $1080 \mathrm{~nm}$. Spectrophotometric measurements were taken three times, and the data were averaged. A Horiba Scientific Fluorolog steady-state spectrofluorometer was employed to quantify emission. Excitation wavelengths at $4 \mathrm{~nm}$ intervals were used between 300 and $750 \mathrm{~nm}$ to characterize the fluorescence between 300 and $1150 \mathrm{~nm}$, a range chosen due to the limit of the machine's photodetectors. The

Table 1 Correspondence table for Luxexcel 3-D printed lenses and their geometrically equivalent glass lenses obtained from Edmund optics (EO) or Thorlabs (TL).

\begin{tabular}{|c|c|c|c|c|}
\hline $\begin{array}{l}\text { Edmund optics/ } \\
\text { Thorlabs } \\
\text { lens ID }\end{array}$ & $\begin{array}{l}\text { Luxexcel } \\
\text { lens ID }\end{array}$ & $\begin{array}{c}\text { Diameter } \\
(\mathrm{mm})\end{array}$ & $\begin{array}{l}\text { Nominal } \\
\text { focal } \\
\text { length } \\
(\mathrm{mm})\end{array}$ & $\begin{array}{c}\text { Nominal } \\
\text { radius of } \\
\text { curvature } \\
(\mathrm{mm})\end{array}$ \\
\hline 32479 (EO) & A_01 & 25 & 75 & 38.76 \\
\hline 32962 (EO) & A_02 & 20 & 60 & 31.01 \\
\hline 32974 (EO) & A_03 & 50 & 150 & 77.52 \\
\hline LA1207 (TL) & B_01 & 12.7 & 100 & 51.5 \\
\hline LA1304 (TL) & B_02 & 12.7 & 40 & 20.6 \\
\hline 63536 (EO) & BiConvexLens_01 & 10 & 50 & 14.8 \\
\hline
\end{tabular}




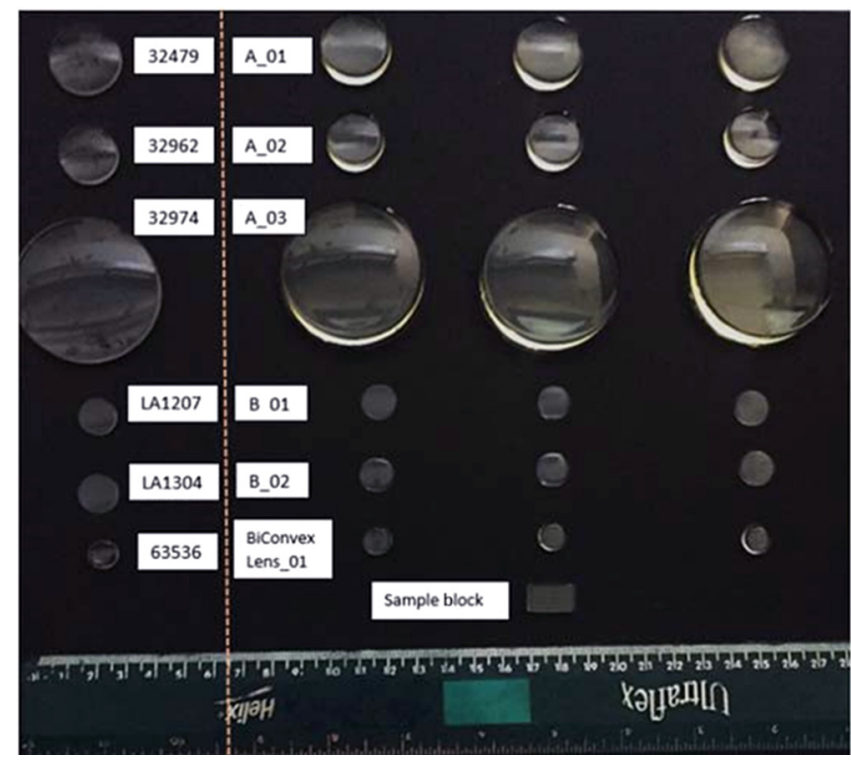

Fig. 1 Image of all tested glass lenses and geometrically equivalent Luxexcel lenses. The dashed line separates the glass lenses (left) with their corresponding 3-D printed prototypes (right). The sample block used for material characterization is shown below the Luxexcel lenses.

scan was run three times, and the results were averaged. We also tested birefringence for all 3-D printed lenses by placing them between two linear polarizers. No visible birefringence was observed for any of the tested Luxexcel lenses.

White-light interferometry measurements were taken to characterize surface roughness ${ }^{33,34}$ using a Zygo NewView 5000 (Zygo, Connecticut). Images of the surface were acquired using a $10 \times$ magnification Mirau objective with a $1.3 \times$ zoom. All roughness measurements were taken across a $0.22 \mathrm{~mm}^{2}$ surface area $(0.55 \mathrm{~mm} \times 0.4 \mathrm{~mm})$. Using the Zygo "micro.app" software, a $20 \mu \mathrm{m}$ vertical scan was performed to measure the surface profile. Zygo built-in least square interpolation routines were used to measure the radius of curvature of each tested lens. The radius of curvature was then subtracted from the measured topographic profile, and a $2.51 \mu \mathrm{m}$ low-pass filter was used to remove noise. The following properties were assessed for each lens for data set with removed spherical component: peak-to-valley $(\mathrm{P}-\mathrm{V})$, root mean square (RMS), and roughness average (Ra). Several measurements were taken for each lens while fine-tuning the mounting of the lens. Measurements were recorded once the PV, RMS, and Ra were minimized after making the appropriate adjustments.

A Zygo PTI 250 Fizeau interferometer was applied to assess the surface figure. ${ }^{33}$ All measurements were acquired using an $F$ /\# 4.8 reference sphere with identical data acquisition and data postprocessing settings. Measurements were taken at the confocal position for each Luxexcel lens as well as the equivalent glass lens. A custom, fixed mask was created to assure that measurements were taken over the same surface area for each lens. The diameter of the circular mask was roughly $10.5 \mathrm{~mm}$. An exception was made for the smallest lens, 63536/BiConvexLens_01, as we needed a reduced mask $(9 \mathrm{~mm})$ to remove fringe pattern artifacts observed at the edges of the clear aperture. The position of each of the tested lenses was adjusted based on the interference pattern, and we manually removed the $z$-axis offset by adjusting the axial position of the measurement head. Tilt was controlled by applying corrections to a tilttip stage driven by micrometer screws. Residual $z$-offset and tilt were removed by the interferometer built-in software. $\mathrm{P}-\mathrm{V}, \mathrm{RMS}$, astigmatism magnitude, and coma magnitude were computed for each lens. Fine tuning was again performed to minimize PTV and RMS for each lens, and these results were recorded. We also took radius of curvature measurements using the Fizeau interferometer. In addition, the radius of curvature of each lens was measured by assessing the difference between the cat's-eye and confocal positions of the measurement head. The $z$-location of the measurement head was provided by an internal linear gauge system that was accessed through MetroPro application "RadScalePTI.app." Due to small variances in the $z$-location traveled as determined by the MetroPro software, radius of curvature measurements were taken three times for each lens and averaged after the mounting of the lenses were adjusted appropriately.

An optical resolution of a test lens was measured using a 1951 USAF resolution target. The 1951 USAF resolution target was illuminated by a white-light source and imaged by the tested lens. Conjugates were set to provide in $1 \times$ magnification. Images of the target were recorded by an 8-bit, monochromatic DMX 72BUC01 CCD camera (The Imaging Source, North Charlotte). To avoid influence of uncorrected chromatic aberrations (all tested lenses were singlets), a $650 \mathrm{~nm}$ narrowband interference filter was used directly behind the illumination source. The imaging system was constructed exclusively from a single, tested lens to avoid degradation of performance due to imperfections of additional components. Since wavefront aberrations are aperture-dependent, we repeated measurement four times for each lens with different arbitrarily selected diameters of a tunable diaphragm that was placed in front of the tested lens. We hypothesize that, at full aperture, the performance of tested lenses is limited by aberrations rather than the diffractive effect. Thus, reduction of the clear aperture results in an improvement of performance up to the point where size of the aperture becomes so small that diffractive effects will dominate.

Material uniformity can also contribute to the performance of an optical component. We created a software model for our system to confirm that the difference in performance could be explained by the surface quality of the lenses. We used Zernike polynomials to approximate the shape of the test lens used in our $1 \times$ magnification imaging test. Zernike polynomial coefficients were interpolated using data from the Fizeau interferometer. The convex surface of the lens was measured using the $F$ /\# 4.8 reference sphere, and the plano surface of the lens was measured using a reference flat. Results obtained from Fizeau interferometer were used to fit the first 16 coefficients of the Zernike polynomials. We modeled our test lens in Zemax ${ }^{\circledR}$ (Radiant Zemax $^{\circledR}$, Redmond, Washington) to measure the theoretical performance. The theoretical performance of the test lens was calculated with measured Zernike polynomials used to approximate the actual surface quality. From this, we took the software-calculated theoretical limit of our test lens in $1 \times$ magnification and compared that with the data we acquired from our imaging experiment to confirm that 
the surface quality could directly explain the theoretical limits of the Luxexcel lenses.

\section{Results}

\subsection{Material Characterization}

We have measured the following properties of Luxexcel Opticlear $^{\circledR}$ optical material: refractive index, transmission, and autofluorescence. For reference purposes, all below measurements were repeated on an in-house sample of PMMA. PMMA is commonly used to fabricate plastic optical components as it has a refractive index and Abbe number similar to BK7 glass. Refractive index data for both the Luxexcel polymer and PMMA are displayed in Fig. 2. The nominal refractive index of the Luxexcel Opticlear ${ }^{\circledR}$ material was obtained from the Luxexcel website ${ }^{35}$ and is plotted with dots. Measured averaged values of the refractive index together with error bars are plotted with triangles. The refractive index data for the reference in-house sample of a PMMA are plotted with rectangles together with corresponding error bars. The refractive index of the Luxexcel material was found to be about $2 \%$ higher than PMMA across all tested wavelengths and about $1 \%$ lower than the nominal value reported by the manufacturer. Our measurements of refractive index were limited to the spectral range of 486 to $656 \mathrm{~nm}$ by the available set of narrowband interference filters compatible with the Atago refractometer.

The profile of transmission was observed across wavelengths of 300 to $1100 \mathrm{~nm}$ as these were the limits of the Varian Cary $50 \mathrm{UV}$-Vis spectrophotometer. The data for PMMA and Luxexcel Opticlear ${ }^{\circledR}$ material are presented in Fig. 3, and transmission curves for Luxexcel and PMMA are marked with blue dots and orange diamonds, respectively. The data display the raw transmission measurements for both materials, including Fresnel losses. Measured transmission was over $80 \%$ at wavelengths above $445 \mathrm{~nm}$ for PMMA and above $450 \mathrm{~nm}$ for Luxexcel material. Transmission in the UVA band rose from $5 \%$ at $320 \mathrm{~nm}$ to $35 \%$ at $400 \mathrm{~nm}$, while transmission of 300 to $315 \mathrm{~nm}$ light in the UVB band remained relatively constant at $5 \%$.

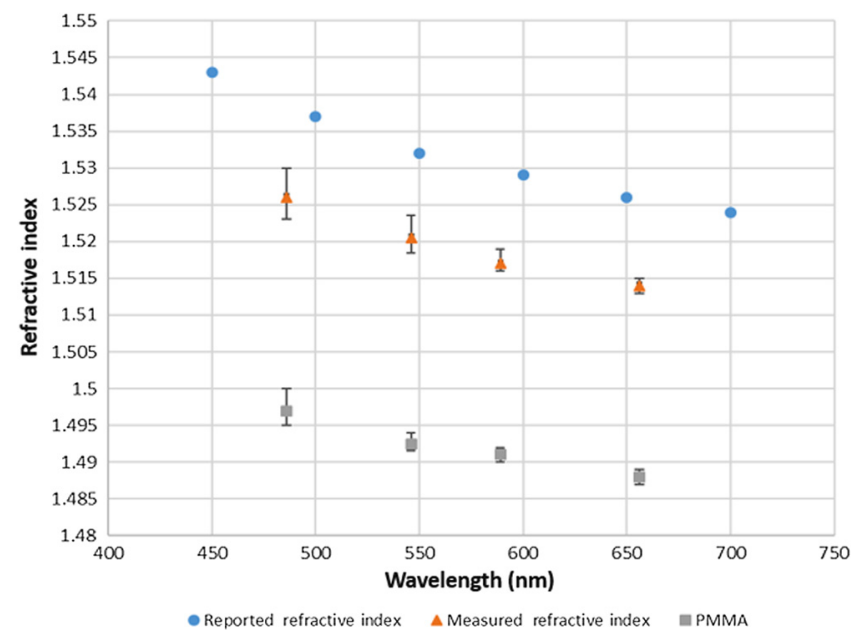

Fig. 2 Measured refractive index of Luxexcel Opticlear ${ }^{\circledR}$ material plotted across visible light spectrum. The blue dots represent the nominal values reported by Luxexcel. The orange triangles display measured results for Luxexcel Opticlear ${ }^{\circledR}$. The gray squares represent data points measured for in-house PMMA sample.

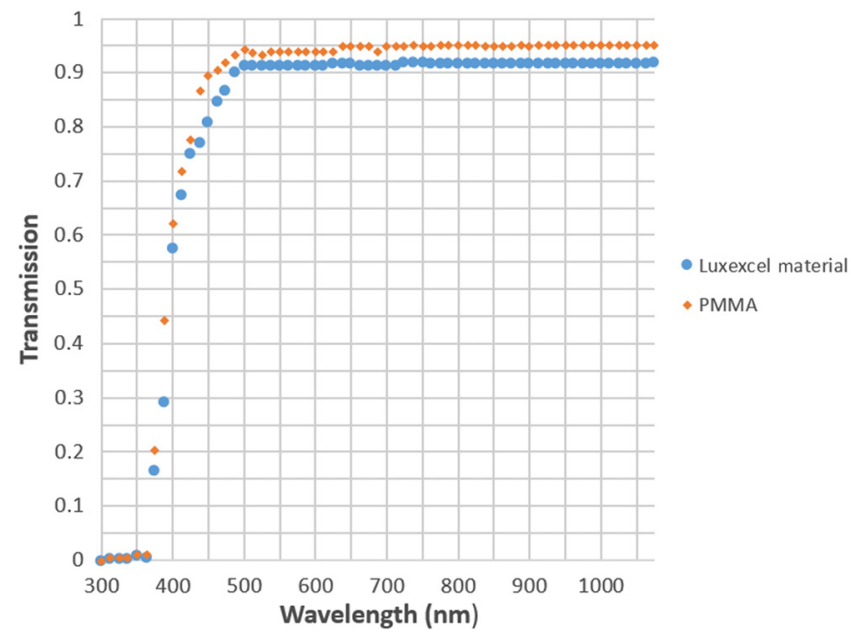

Fig. 3 Plot of transmission versus wavelength for Luxexcel Opticlear ${ }^{\circledR}$ polymer (blue circles) and reference in-house PMMA sample (orange diamonds).

On average, transmission was only $4 \%$ higher for PMMA, a small difference that makes both materials similar in terms of light transmission properties.

The same samples used in the spectrophotometric measurements were used to characterize material autofluorescence. Each sample was illuminated with excitation wavelengths of 300 to $750 \mathrm{~nm}$, and emission was measured between 300 and $1150 \mathrm{~nm}$. Both ranges were hardware limited by the Horiba Scientific Fluorolog spectrofluorometer. The data are displayed in Fig. 4. The subplot 4(a) shows excitation-emission matrix for the PMMA sample. The excitation-emission matrix for Luxexcel Opticlear ${ }^{\circledR}$ polymer is presented in Fig. 4(b). Both two-dimensional (2-D) plots have logarithmic color scales and unified color bar ranges for visualization purposes. As shown in Figure 4(a), the PMMA sample exhibits weak autofluorescence in the excitation range of 350 to $400 \mathrm{~nm}$. On the other hand, the Opticlear ${ }^{\circledR}$ polymer has broadband emission in visible spectrum when excited in the wavelength range of 350 to $500 \mathrm{~nm}$. Stokes shifts for most fluorophores are typically on the order of tens of nm, which suggests nonfluorescent applications of the 3-D printed plastic.

\subsection{Roughness and Radii of Curvature Measurements}

Using white-light interferometry, we measured the radius of curvature, P-V, RMS, and Ra for the glass reference lenses and the Luxexcel lenses. Table 2 displays the averaged results where all values were rounded to the nearest nanometer. Raw data for each lens can be seen in Table 3. Minimum, maximum, and average values are used to show range and variance within the three 3-D printed samples of each design. While radii of curvature were found to be within $0.5 \%$ of the nominal value for most of the glass lenses, the radius of curvature for the 3-D printed prototypes deviated from nominal values anywhere from $0.2 \%$ to $13 \%$. In addition, surface irregularities quantified by P-V and RMS deviation were an order of magnitude higher for the 3-D printed prototypes as compared with reference glass lenses. The roughness values of 3-D printed lenses, however, are comparable with the roughness of plastic lenses fabricated with injection 

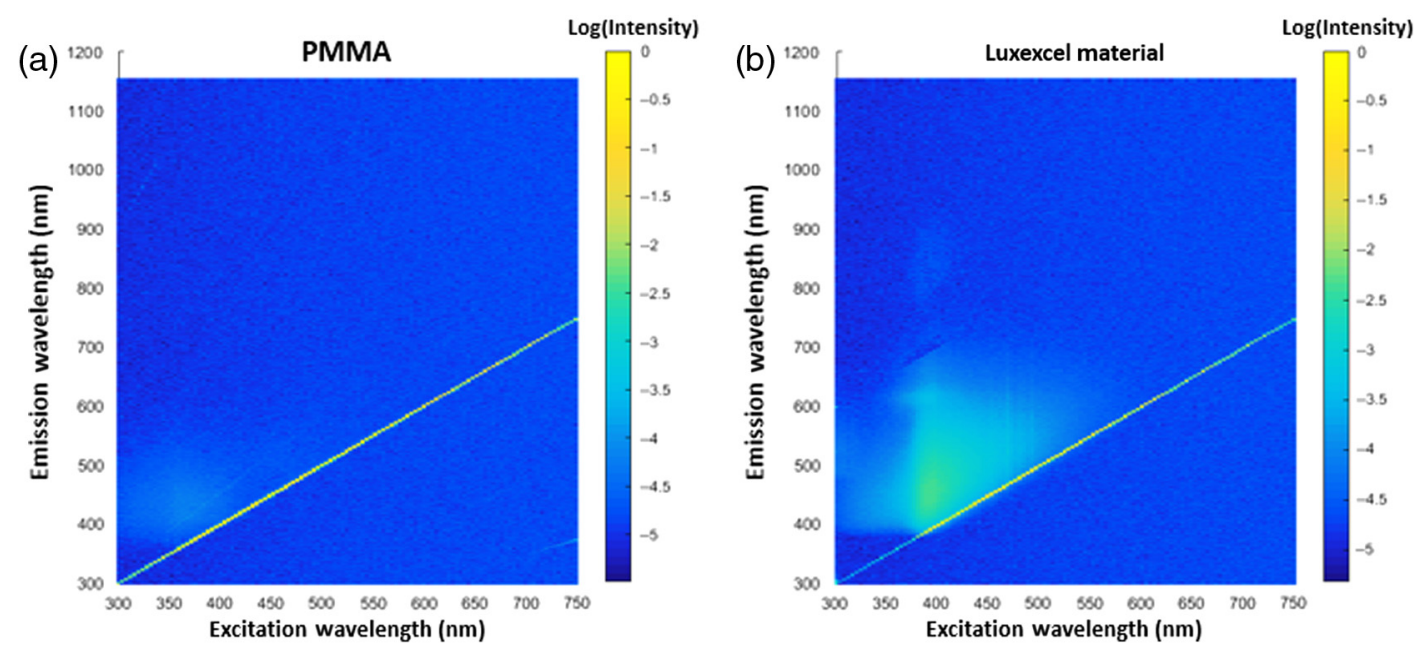

Fig. 4 Excitation-emission matrices for (a) PMMA and (b) Luxexcel Opticlear ${ }^{\circledR}$.

Table 2 White-light interferometry data for 3-D printed lenses and glass lenses. For 3-D printed lenses, the best-and worst-performing lens in each category from each set of 3 is shown along with the average values.

\begin{tabular}{|c|c|c|c|c|c|c|c|c|c|c|c|c|c|}
\hline \multirow[b]{2}{*}{ Lens ID } & \multirow{2}{*}{$\begin{array}{l}\text { Nominal radius of } \\
\text { curvature }(\mathrm{mm})\end{array}$} & \multicolumn{3}{|c|}{$\begin{array}{l}\text { Software-calculated } \\
\text { radius of curvature } \\
(\mathrm{mm})\end{array}$} & \multicolumn{3}{|c|}{$\mathrm{P}-\mathrm{V}(\mathrm{nm})$} & \multicolumn{3}{|c|}{$\mathrm{RMS}(\mathrm{nm})$} & \multicolumn{3}{|c|}{$\mathrm{Ra}(\mathrm{nm})$} \\
\hline & & Min. & Max. & Avg. & Min. & Max. & Avg. & Min. & Max. & Avg. & Min. & Max. & Avg \\
\hline 32479 & 38.76 & & & 38.81 & & & 5 & & & 1 & & & 1 \\
\hline A_01 & & 33.94 & 34.60 & 34.32 & 96 & 125 & 111 & 20 & 23 & 22 & 16 & 18 & 17 \\
\hline 32962 & 31.01 & & & 31.01 & & & 7 & & & 1 & & & 1 \\
\hline A_02 & & 30.99 & 31.14 & 31.08 & 27 & 39 & 32 & 6 & 11 & 9 & 5 & 10 & 8 \\
\hline 32974 & 77.52 & & & 77.34 & & & 7 & & & 1 & & & 1 \\
\hline A_03 & & 63.89 & 74.27 & 70.60 & 48 & 68 & 55 & 13 & 19 & 15 & 11 & 16 & 13 \\
\hline LA1207 & 51.5 & & & 51.47 & & & 5 & & & 1 & & & 1 \\
\hline B_01 & & 44.84 & 45.79 & 45.19 & 43 & 46 & 44 & 8 & 11 & 10 & 6 & 10 & 8 \\
\hline LA1304 & 20.6 & & & 20.58 & & & 7 & & & 1 & & & 1 \\
\hline B_02 & & 19.19 & 19.32 & 19.26 & 44 & 108 & 66 & 8 & 20 & 13 & 6 & 16 & 10 \\
\hline 63536 & 14.88 & & & 14.96 & & & 10 & & & 2 & & & 2 \\
\hline BiConvexLens_01 & & 14.72 & 14.77 & 14.73 & 37 & 85 & 61 & 6 & 18 & 13 & 6 & 15 & 11 \\
\hline
\end{tabular}

molded processes, which are on the order of single $\mathrm{nm}$ for state of the art and $\sim 10 \mathrm{~nm}$ for "normal" quality production processes. $^{4}$

Figure 5(a) displays a 2-D surface profile of a Thorlabs LA1207 lens, and Fig. 5(b) shows its geometric equivalent, a Luxexcel B_01 lens. This pair of lenses was selected because Luxexcel B_01 has an average P-V and RMS deviation compared with the tested population. In both 2-D maps presented in Fig. 5, the measured radius of curvature has been removed. For the LA1207 lens and its 3-D printed equivalent, deviations from sphericity were within $11 \mathrm{~nm}$ for the glass lens and within $169 \mathrm{~nm}$ for the plastic lens. The scale bars of 2-D plots in Figs. 5(a) and 5(b) have different ranges to visualize a full dynamic range of a respective data set. The inset in Fig. 5(a) has a color bar range identical to the color bar in Fig. 5(b) to assist in comparative and qualitative evaluation of both lenses.

\subsection{Form Measurements}

Wavefront deformations caused by irregularity in lens surfaces were evaluated using a Fizeau interferometer. Basic surface figure statistics are presented in Tables 4 and 5 . Averaged radii of curvature, RMS, and P-V are given in Table 4. Averaged astigmatism and coma magnitudes are displayed in Table 5. All numerical values measured in waves 
Table 3 White-light interferometry data comparison for 3-D printed lenses and glass lenses.

\begin{tabular}{|c|c|c|c|c|c|}
\hline Lenses ID & $\begin{array}{l}\text { Nominal radius } \\
\text { of curvature } \\
(\mathrm{mm})\end{array}$ & $\begin{array}{l}\text { Software- } \\
\text { calculated } \\
\text { radius of } \\
\text { curvature }(\mathrm{mm})\end{array}$ & $\begin{array}{l}\mathrm{P}-\mathrm{V} \\
(\mathrm{nm})\end{array}$ & $\begin{array}{l}\text { RMS } \\
(\mathrm{nm})\end{array}$ & $\begin{array}{c}\mathrm{Ra} \\
(\mathrm{nm})\end{array}$ \\
\hline 32479 & 38.76 & 38.81 & 5 & 1 & 1 \\
\hline A_01 (1) & & 33.94 & 125 & 20 & 16 \\
\hline A_01 (2) & & 34.60 & 112 & 22 & 18 \\
\hline A_01 (3) & & 34.43 & 96 & 23 & 18 \\
\hline 32963 & 31.01 & 31.01 & 7 & 1 & 1 \\
\hline A_02 (1) & & 30.99 & 27 & 6 & 5 \\
\hline A_02 (2) & & 31.10 & 39 & 11 & 10 \\
\hline A_02 (3) & & 31.14 & 30 & 11 & 10 \\
\hline 3297477 & 77.52 & 77.34 & 7 & 1 & 1 \\
\hline A_03 (1) & & 63.89 & 68 & 19 & 16 \\
\hline A_03 (2) & & 74.27 & 50 & 13 & 11 \\
\hline A_03 (3) & & 73.65 & 48 & 14 & 12 \\
\hline LA1207 & 51.5 & 51.47 & 5 & 1 & 1 \\
\hline B_01 (1) & & 44.95 & 43 & 8 & 6 \\
\hline B_01 (2) & & 45.79 & 44 & 11 & 10 \\
\hline B_01 (3) & & 44.84 & 46 & 10 & 9 \\
\hline LA1304 & 20.6 & 20.58 & 7 & 1 & 1 \\
\hline B_02 (1) & & 19.32 & 108 & 20 & 16 \\
\hline B_02 (2) & & 19.19 & 44 & 8 & 6 \\
\hline B_02 (3) & & 19.28 & 47 & 10 & 9 \\
\hline 63536 & 14.88 & 14.96 & 10 & 2 & 2 \\
\hline $\begin{array}{l}\text { BiConvex } \\
\text { Lens_01 (1) }\end{array}$ & & 14.74 & 85 & 18 & 15 \\
\hline $\begin{array}{l}\text { BiConvex } \\
\text { Lens_01 (2) }\end{array}$ & & 14.72 & 37 & 6 & 6 \\
\hline $\begin{array}{l}\text { BiConvex } \\
\text { Lens_01 (3) }\end{array}$ & & 14.77 & 62 & 14 & 12 \\
\hline
\end{tabular}

were rounded to the nearest thousandth, and all results presented in units of length were rounded to the nearest hundredth of a millimeter. Raw data for both tables can be found in Table 6. Minimum, maximum, and average values are used to show range and variance for each set of measurements for both the glass lenses and their corresponding 3-D printed prototypes. In general, Luxexcel lenses exhibited larger magnitudes of aberrations than their geometric equivalents made from glass. The magnitudes of coma and astigmatism for the 3-D printed lenses were one to two orders higher than their glass counterparts. Lens B_01 had the smallest measured magnitudes of coma and astigmatism, whereas lens A_01 had the largest measured combined magnitudes of aberrations. RMS and P-V metrics were also smallest for the B_01 lens. Its RMS was however 21 times higher and P-V was 15 times higher than the corresponding glass lens of the same geometry. Similar in terms of aberrations magnitude, lens B_02 has an identical 12.7-mm diameter. Data from Tables 4 and 5 suggest that the magnitude of aberrations, together with P-V and RMS, are roughly proportional to the diameter. We hypothesize that this may be due to $3-\mathrm{D}$ printing machine positioning tolerances and/or process-specific injection/curing parameters. The smallest tested lens, BiConvexLens_01, had a diameter of $10 \mathrm{~mm}$ and high magnitudes of aberrations together with large values of P-V and RMS. The biconvex lens does not appear to follow the trend we see among the other plano-convex lenses, in which aberrations increase with lens diameter. We attribute this observation to a Luxexcel-specific manufacturing/assembly process. Luxexcel-specific 3-D printing technology requires assembly of nonplano lenses from any combination of plano-convex and plano-concave components, and this assembly process is likely responsible for the increased magnitude of aberrations.

Example of 2-D wavefront plots for Edmund Optics 32962 and its 3-D printed equivalent are presented in Figs. 6(a) and 6(b), respectively. The Luxexcel A_02 lens was selected because it has representative performance for a population of all tested 3-D printed lenses. It has P-V and RMS deviation of 4.962 and 0.760 waves, respectively. As shown in Fig. 6(a), wavefront errors of the reference lens were within a range of 0.08 waves, while corresponding 3-D printed lens surface deviations, shown in Fig. 6(b), were within 4.7 waves. The insert in Fig. 6(a) presents reference glass lens wavefront deformations color coded in the range identical to the plot given in Fig. 6(b) to aid in visual comparison between both components. (a)

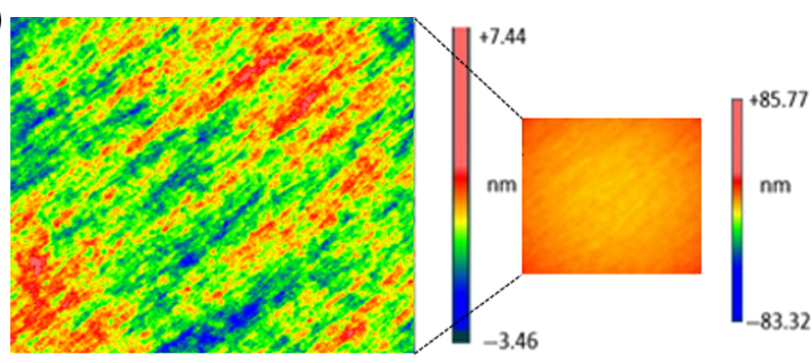

(b)

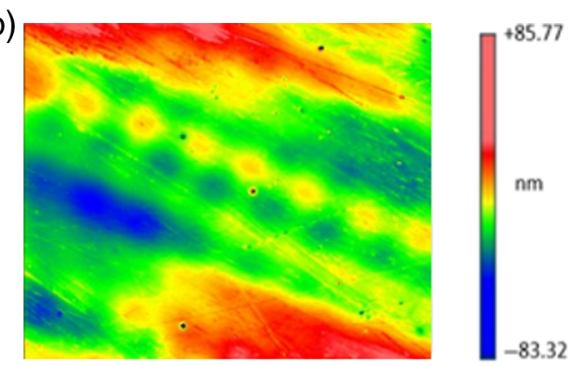

Fig. 5 White-light interferometry measured surface profiles of (a) Thorlabs lens LA1207 and nominally identical 3-D printed B_01 lens (b). Color bar ranges for both images are different to visualize different dynamic ranges of both data sets. For visual comparison purposes, the surface plot of glass lens shown in (a) is presented in the inset with color bar scale range identical to subfigure (b). 
Table 4 Statistics for radius of curvature, P-V, and RMS measured using a Fizeau interferometer. Averages shown are for 3-D prototypes as there were three samples of each. For radius of curvature measurement, multiple measurements were also taken and averaged due to small variance in the measured movement of the $z$-position as determined by the software.

\begin{tabular}{|c|c|c|c|c|c|c|c|c|c|c|}
\hline \multirow[b]{2}{*}{ Lens ID } & \multirow{2}{*}{$\begin{array}{l}\text { Nominal radius of } \\
\text { curvature }(\mathrm{mm})\end{array}$} & \multicolumn{3}{|c|}{$\begin{array}{c}\text { Fizeau-measured radius of } \\
\text { curvature }(\mathrm{mm})\end{array}$} & \multicolumn{3}{|c|}{ P-V (wave) } & \multicolumn{3}{|c|}{ RMS (wave) } \\
\hline & & Min. & Max. & Avg. & Min. & Max. & Avg. & Min. & Max. & Avg. \\
\hline 32479 & 38.76 & 38.77 & 38.77 & 38.77 & & & 0.637 & & 0.012 & \\
\hline A_01 & & 34.20 & 34.87 & 34.60 & 7.224 & 8.074 & 7.717 & 1.620 & 1.744 & 1.698 \\
\hline 32962 & 31.01 & 31.02 & 31.02 & 31.02 & & & 0.080 & & 0.007 & \\
\hline A_02 & & 30.88 & 31.04 & 30.97 & 4.692 & 12.383 & 7.600 & 0.760 & 2.382 & 1.510 \\
\hline 32974 & 77.52 & 77.46 & 77.46 & 77.46 & & & 0.079 & & 0.008 & \\
\hline A_03 & & 68.35 & 74.10 & 71.91 & 7.169 & 8.236 & 7.554 & 1.620 & 1.652 & 1.680 \\
\hline LA1207 & 51.5 & 51.28 & 51.29 & 51.28 & & & 0.135 & & 0.016 & \\
\hline B_01 & & 44.90 & 45.63 & 45.25 & 1.908 & 2.140 & 2.048 & 0.301 & 0.379 & 0.336 \\
\hline LA1304 & 20.6 & 20.58 & 20.59 & 20.59 & & & 0.140 & & 0.014 & \\
\hline B_02 & & 19.10 & 19.31 & 19.18 & 2.232 & 2.753 & 2.425 & 0.385 & 0.441 & 0.408 \\
\hline 63536 & 14.88 & 14.88 & 14.88 & 14.88 & & & 0.127 & & 0.016 & \\
\hline BiConvexLens_01 & & 14.69 & 14.87 & 14.72 & 7.553 & 8.027 & 7.858 & 1.616 & 1.692 & 1.657 \\
\hline
\end{tabular}

Table 5 Calculated astigmatism and coma magnitudes for 3-D printed polymer and glass lenses.

\begin{tabular}{|c|c|c|c|c|c|c|}
\hline \multirow[b]{2}{*}{ Lens ID } & \multicolumn{3}{|c|}{$\begin{array}{c}\text { Astigmatism } \\
\text { magnitude (wave) }\end{array}$} & \multicolumn{3}{|c|}{$\begin{array}{l}\text { Coma magnitude } \\
\text { (wave) }\end{array}$} \\
\hline & Min. & Max. & Avg. & Min. & Max. & Avg. \\
\hline 32479 & & & 0.011 & & & 0.037 \\
\hline A_01 & 7.755 & 8.434 & 8.169 & 1.016 & 1.231 & 1.097 \\
\hline 32962 & & & 0.012 & & & 0.024 \\
\hline A_02 & 3.554 & 11.695 & 6.538 & 0.876 & 1.112 & 0.999 \\
\hline 32974 & & & 0.024 & & & 0.029 \\
\hline A_03 & 7.743 & 8.575 & 8.078 & 0.432 & 1.041 & 0.766 \\
\hline LA1207 & & & 0.029 & & & 0.008 \\
\hline B_01 & 1.275 & 1.580 & 1.429 & 0.679 & 0.767 & 0.723 \\
\hline \multirow[t]{2}{*}{ LA1304 B_02 } & & & 0.033 & & & 0.016 \\
\hline & 1.481 & 2.389 & 1.907 & 0.545 & 0.767 & 0.664 \\
\hline 63536 & & & 0.034 & & & 0.056 \\
\hline BiConvexLens_01 & 2.223 & 3.022 & 2.656 & 2.678 & 3.022 & 2.858 \\
\hline
\end{tabular}

Surface error profiles were found to be both systematic and random as shown in Fig. 7. Figure 7(a) is a display of the Fizeau surface profile for each of the three A_02 lenses. The error profiles are all qualitatively similar, albeit at different rotational angles. Figure 7(b) presents the surface measurements for the three B_01 lenses, in which one of the B_01 lenses has a surface profile with a shape and features (left) different than the other two lenses of the same type (middle and right). A further analysis of the presence of random error in the manufacturing process would require a higher volume of tested lenses.

\subsection{Imaging Performance}

White-light interferometry data and wavefront aberrations measured using a Fizeau interferometer are sufficient for assessing the quality of an optical component. While wavefront aberrations can be used to infer performance of the optical system, including resolution, we decided to also experimentally measure the resolution of two arbitrarily selected lenses using the 1951 USAF resolution target. For this test, we selected the best-performing 3-D printed lens, B_01, and its glass counterpart LA1207. Resolution was measured at four different diameters of a manually controlled aperture, placed directly in front of the tested lens. Images of 1951 USAF resolution target given by the Thorlabs LA1207 lens are shown in Fig. 8 and corresponding results for the Luxexcel B_01 lens are presented in Fig. 9. In a $1 \times$ magnification configuration, the LA1207 lens reached resolutions of 22.63, 32.0, 45.3, and $50.8 \mathrm{lp} / \mathrm{mm}$ for adjustable aperture diameters of $4,6,8$, and $10 \mathrm{~mm}$, respectively. Under identical conditions, B_01 reached 22.63, 32.0, 32.0, and $28.51 \mathrm{lp} / \mathrm{mm}$ resolution. The resolution of the glass lens rose proportionally with an increase in adjustable aperture diameter. Contrary to this, resolution of the $3-\mathrm{D}$ printed polymer 
Table 6 Fizeau data comparison for Luxexcel lenses against corresponding Edmund optics/Thorlabs lenses. Radius of curvature measurements were taken three times for each lens.

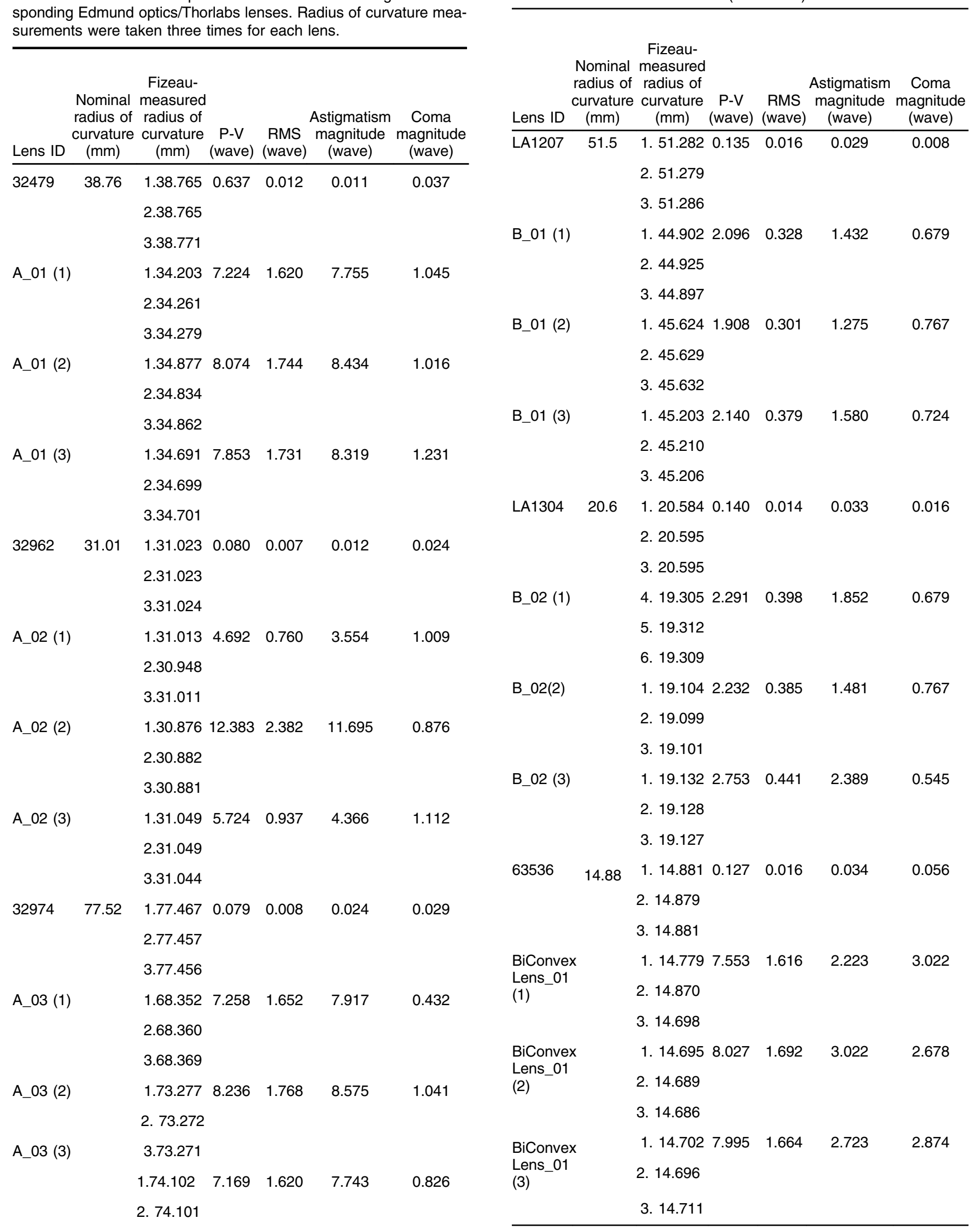

Table 6 (Continued).

3. 74.098 
(a)

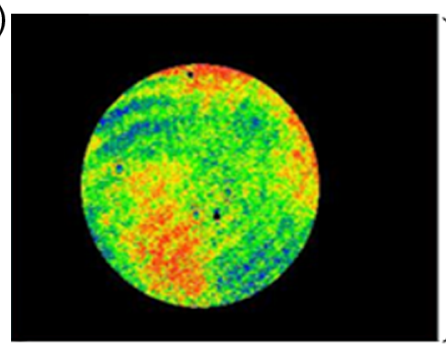

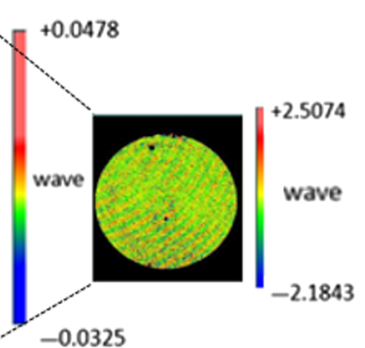

(b)
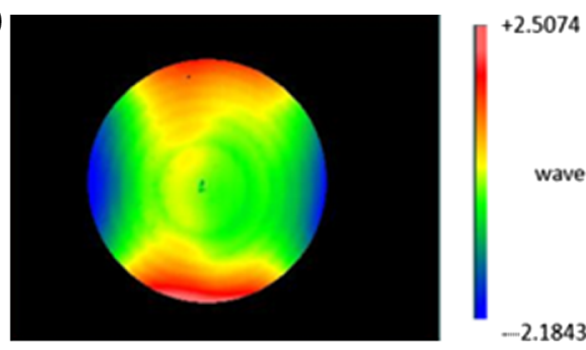

Fig. 6 (a) 2-D plot of the wavefront error of the Edmund optics 32962 lens and (b) equivalent 3-D printed A_02 lens. Color bars in subfigures have different magnitudes to visualize full dynamic ranges of both profiles. Inset in Fig. 6(a) has color bar scale identical to Fig. 6(b) to enable qualitative comparison between both lenses.

(a)

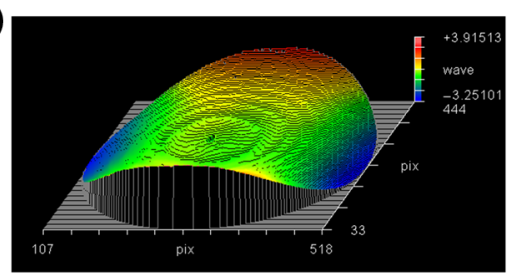

(b)

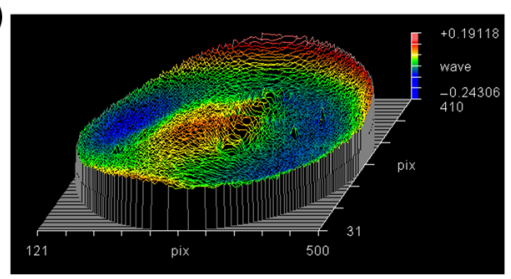

A_02

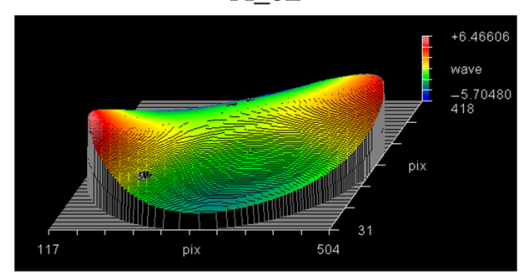

B_01

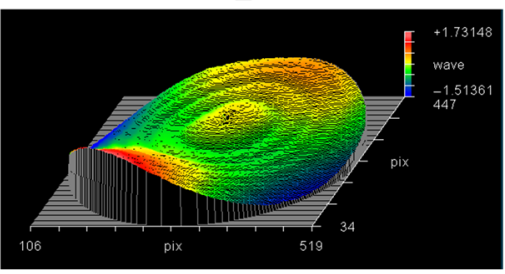

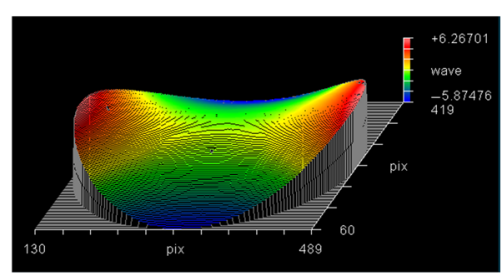

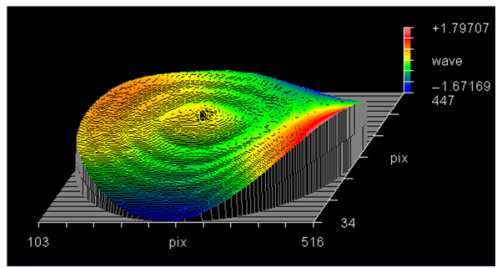

Fig. 7 Presence of systematic and random error in the 3-D printed lenses. The top row (a) shows lens A_02, where all lenses have a similar error profile at different rotational angles. The bottom row (b) shows lens B_01, where the leftmost lens has a different surface error profile than the middle and right lenses. Thus, some error in the manufacturing process is random.

lens was highest for a midrange aperture between $6 \mathrm{~mm}$ and $8 \mathrm{~mm}$. In general, the resolution of an optical system working in finite conjugates depends on the balance between wavefront aberration and system NA. For well-corrected systems, the resolution depends exclusively on NA and can be described by the Rayleigh criterion. In the case of aberrated systems, the resolution will depend on the balance between aperture size and wavefront aberrations. Since wavefront deformations are field dependent and usually decrease together with reductions of the clear aperture, we speculate that the best performance of the plastic lens for a 6 to $8 \mathrm{~mm}$ diaphragm was due to the optimal balance between aberrations and the input beam NA. The increase of aperture diameter should theoretically improve resolution of the polymer lens, but, due do surface irregularities, the resolution decreased and the system performance was effectively limited by aberrations rather than diffractionlimited. Please note that in support of this claim, at an aperture of $10 \mathrm{~mm}$, the image of the 1951 USAF is brightest and has lowest contrast among all images recorded using a polymer lens. Overall, the Luxexcel lens resolution was below its glass equivalent for all measured clear aperture settings, but it was quite acceptable for applications that do not emphasize resolution, such as low-resolution imaging and illumination systems.

\subsection{Effect of Surface Error on Performance}

Figure 10 displays the results of our software model used to characterize the theoretical limit of lens B_01. Spot diagrams for each of the aperture diagrams used in the imaging experiment are shown in Fig. 9. The performance of B_01 is shown with its surface shape approximated using the first 16 Zernike polynomials measured on the test lens on the Fizeau interferometer. Spot diagrams and airy disks are shown at the center of the field of view (FOV) (left) and at distances of 1.75 and $2.5 \mathrm{~mm}$ away from the center of the FOV for each aperture size. While we show these three different axis points, experimental verification was based on results from the on-axis simulation shown in the left column of Fig. 10 as the smallest resolvable resolution targets were placed in the center of the FOV of the imaging system. When the RMS radius is lower than the airy disk radius, the system is diffraction-limited, and the airy disk radius will determine the resolution limit of the system. However, when the RMS radius is larger, the system will be limited by aberrations, and the RMS radius can be used to approximate the theoretical resolution limit. The theoretical performance was compared with the actual performance of the lens measured at a full aperture depicted in Figure 9. For the aperture sizes shown in the top row of Fig. 10, the system is diffraction limited: a system with 

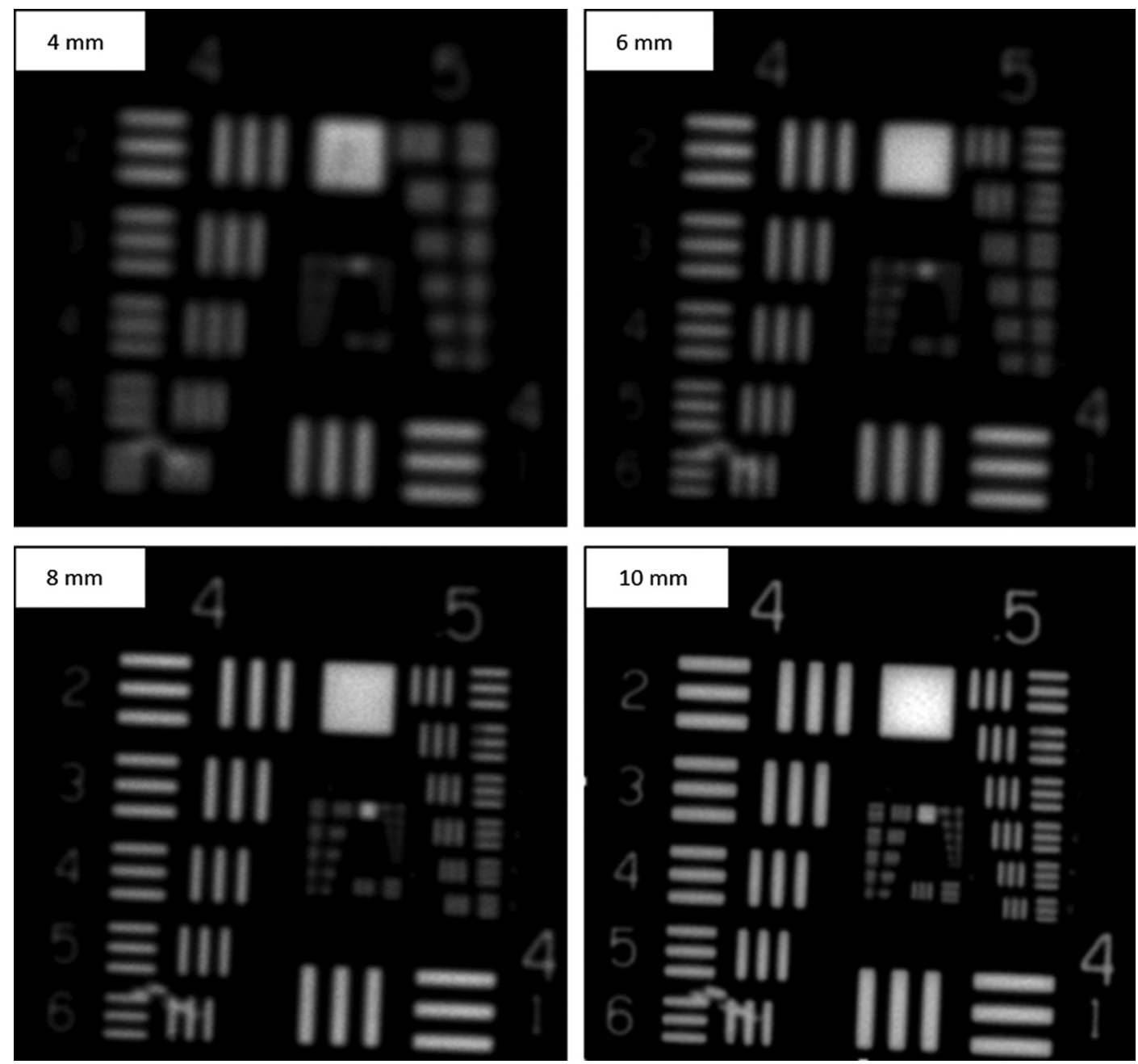

Fig. 8 Images of the 1951 USAF resolution target recorded by the Thorlabs lens LA1207, in 1× magnification configuration. Diameter of the adjustable aperture is given in the insets.

a clear aperture reduced to $4 \mathrm{~mm}$ has a resolution limit of $24.35 \mathrm{lp} / \mathrm{mm}$, and a system with a $6 \mathrm{~mm}$ aperture has a resolution limit of $36.59 \mathrm{lp} / \mathrm{mm}$. For the aperture sizes in the bottom row of Fig. 10, the theoretical performance is aberration-limited, where on-axis performance is determined by the RMS radius listed in the left column. The on-axis theoretical performance is as follows: when the system has a reduced clear aperture of $8 \mathrm{~mm}$ the resolution is $44.27 \mathrm{lp} / \mathrm{mm}$, and, at a full aperture of $10 \mathrm{~mm}$, the resolution limit is $31.17 \mathrm{lp} / \mathrm{mm}$ at the center of the FOV and $23.94 \mathrm{lp} / \mathrm{mm}$. Strehl ratios were measured as 0.941 for $4 \mathrm{~mm}$ aperture, 0.777 for $6 \mathrm{~mm}$ aperture, 0.557 for $8 \mathrm{~mm}$ aperture, and 0.424 for $10 \mathrm{~mm}$ aperture. Given that resolution using a USAF target is discrete, we should expect the resolution limit to be group 4, element 4 for a $4 \mathrm{~mm}$ aperture; group 5, element 2 for a $6 \mathrm{~mm}$ aperture; group 5, element 3 for an $8 \mathrm{~mm}$ aperture; and group 4, element 6 for a $10 \mathrm{~mm}$ aperture. These theoretical values are all within 1 to 2 elements of the resolution limits that we report above. This indicates that, while there may be other factors contributing to the performance of these lenses (such as material uniformity), the surface figure dominantly impacts the overall system performance.

\section{Discussion}

We have assessed the roughness, wavefront aberrations, and imaging capabilities of plastic lenses fabricated using a 3-D prototyping technology and compared their quality to geometrically identical state-of-the art glass lenses. Whitelight interferometry results showed that surface roughness of the 3-D printed lenses was between 10 and $20 \mathrm{~nm}(\mathrm{Ra})$ and was one order of magnitude higher than roughness of reference glass equivalents ( $1 \mathrm{~nm} \leq \mathrm{Ra} \leq 2 \mathrm{~nm}$ ). Deviations from sphericity, as measured using the Fizeau interferometer, were higher for the 3-D printed lenses than for glass counterparts. Typically, P-V deviation of the plastic lenses was found to be on the order of a single wave, while the equivalent glass substitutes' P-V was on the level of tenths of the test wavelength. Measured coma and astigmatism magnitudes were two orders of magnitude higher in the cases of the plastic lenses than comparable glass components. The 3-D printed lenses consistently had higher surface figure errors than their glass counterparts. As shown in Fig. 7, the source of error is both random and systematic, though a majority of the errors we observed were systematic. This systematic error is potentially correctable as the state of the technology matures. 

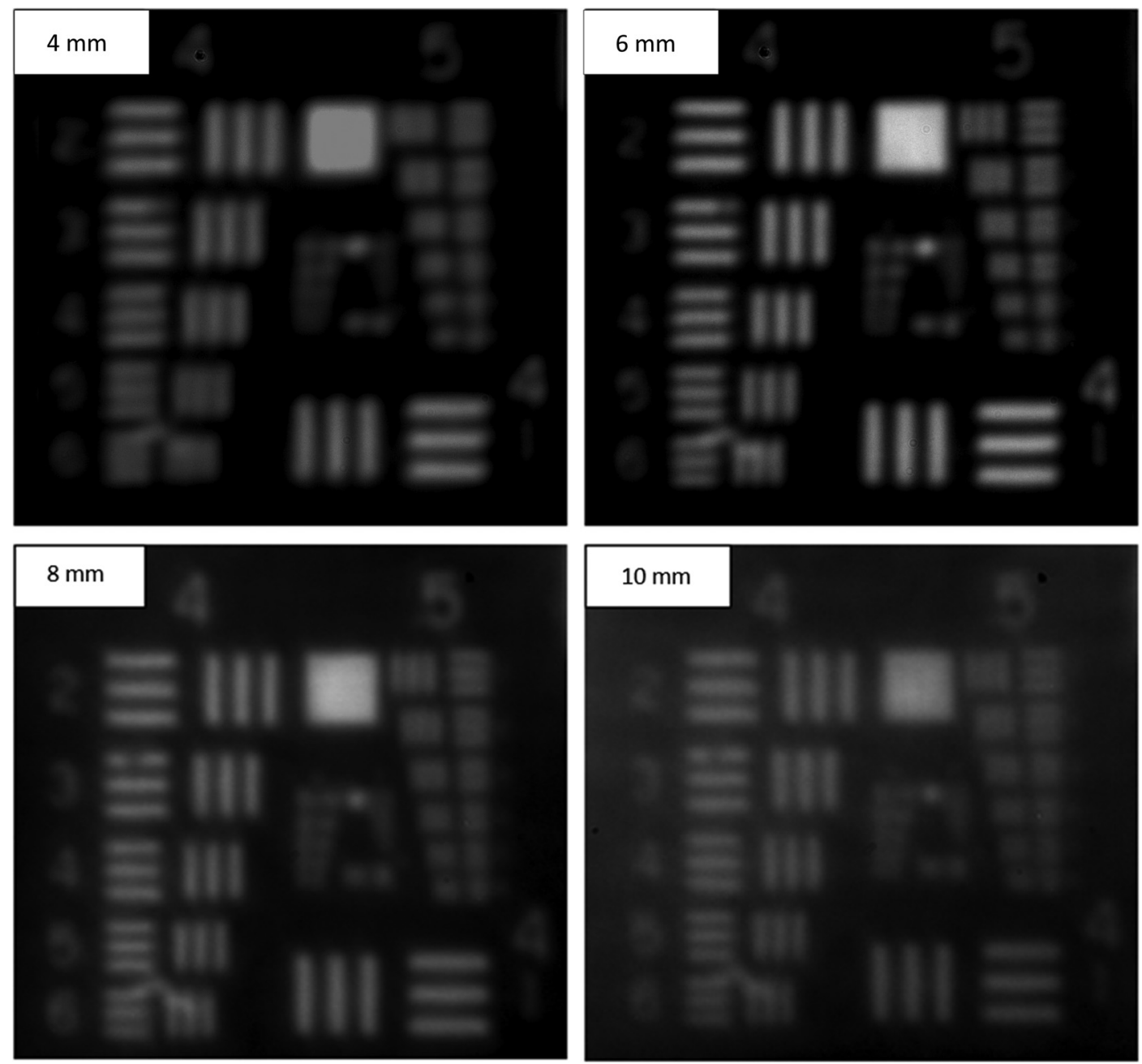

Fig. 9 Images of a 1951 USAF resolution target recorded by the Luxexcel lens B_01, in 1× magnification conjugates. Diameter of the adjustable aperture is given in the insets.

We experimentally measured the performance of an arbitrarily selected 3-D printed lens and compared it with the performance of a nominally identical glass lens. Since 3-D printed lenses had larger magnitudes of aberrations, and their RMS and P-V metrics were one order to two orders of magnitude higher than glass equivalents, our imaging system built using a plastic lens suffered from reduced contrast and had lower resolution. We were able to optimize performance of a plastic lens by adjusting its aperture stop diameter. We found that the resolution of a plastic B_01 lens was maximum when the clear aperture was closed down to about $63 \%$ of the nominal diameter. Since resolution of a lens is a result of balance between theoretically predicted wavefront deformation and aberrations induced by surface irregularities, reduction of clear aperture decreases the theoretically achievable resolution and simultaneously decreases wavefront aberrations. For that reason, for clear apertures below $63 \%$ of a full aperture, the tested plastic lens was primarily limited by the diffraction effects and, for clear apertures above it, system performance was dominated by aberrations induced by lens surface irregularities. Analysis of lens performance versus design parameters suggests that the analyzed 3-D printed process favors smaller lenses that have larger radii of curvature. Measured RMS values together with P-V deformations were highest for the two largest lenses, A_01 and A_03, which had 20- and 50-mm diameters, respectively. The two lenses with the smallest diameters, B_01 and B_02, had the smallest values of P-V and RMS. We have also experimentally measured the index of refraction and transmission of the 3-D-printable Luxexcel Opticlear ${ }^{\circledR}$ material. We found that measured values of $n(\lambda)$ are about $1 \%$ lower than that reported by the manufacturer, which may be attributed to batch to batch variation in optical properties that are typical for many other optical materials including polystyrene and PMMA. It should be noted that refractive index curve of the Opticlear ${ }^{\circledR}$ was very similar to the refractive index curve of the PMMA, albeit the index of refraction for Opticlear ${ }^{\circledR}$ was on average $2 \%$ lower. Transmission of the 3-mm thick block made from Opticlear $^{\circledR}$ was $95 \%$ in the visible part of the electromagnetic spectrum. Spectral transmission curve of the Opticlear ${ }^{\circledR}$ was very similar to transmission curve of the PMMA, albeit the absorption of Opticlear ${ }^{\circledR}$ was about $4 \%$ higher at visible wavelengths and was identical to PMMA in the UVA region (315-400 nm). Tested 3-D printable material exhibited autofluorescent properties in the excitation range of 370 to $550 \mathrm{~nm}$. The emission maximum of the Opticlear ${ }^{\circledR}$ was located between wavelengths of 400 and $550 \mathrm{~nm}$, and the 


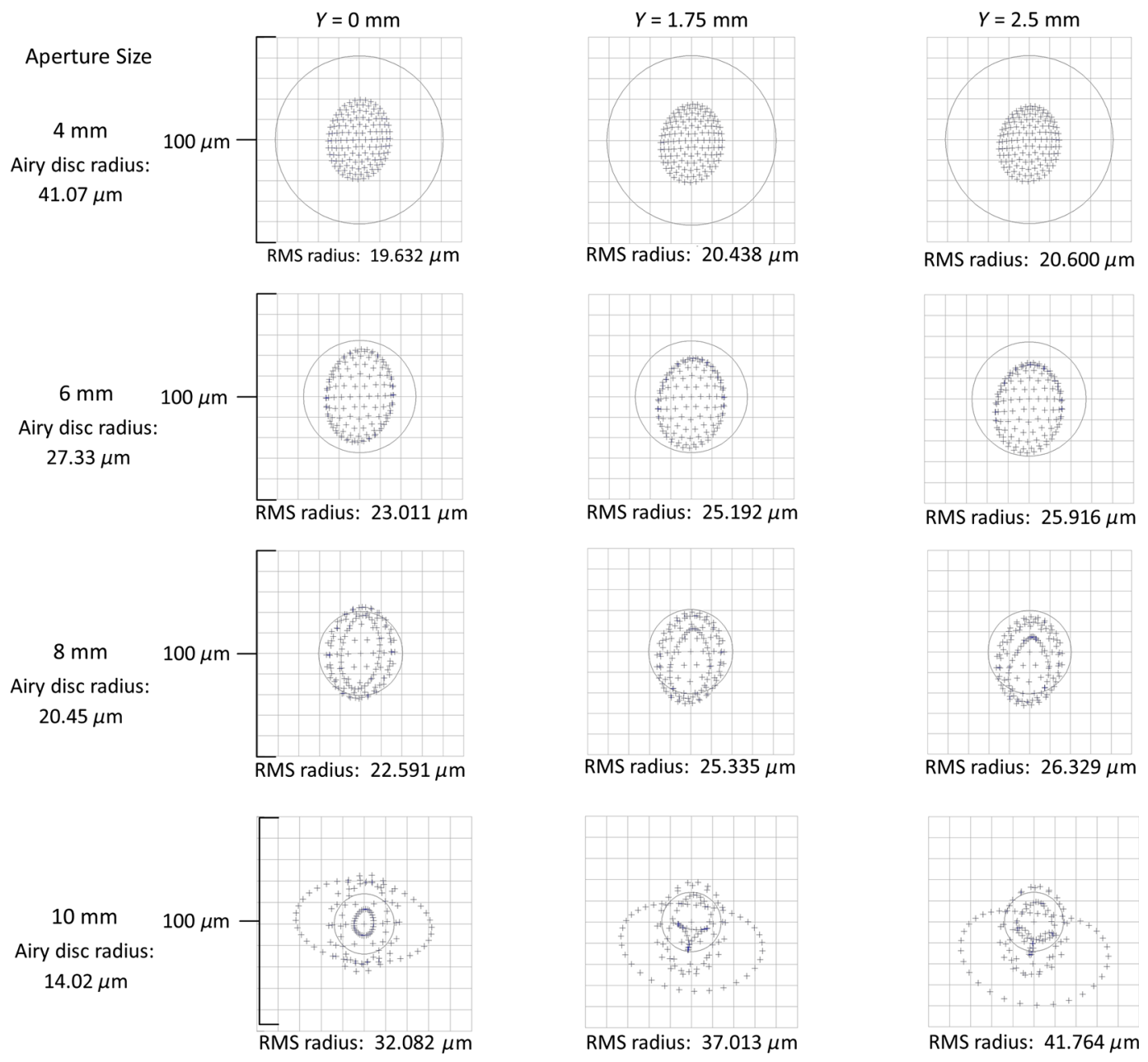

Fig. 10 Performance metrics of lens B_01 modeled in Zemax ${ }^{\circledR}$ for aperture sizes of 4, 6, 8, and $10 \mathrm{~mm}$. Airy disk radius is shown under the aperture sizes on the left. Spot diagrams with RMS radius values are shown at the center of the field of view and at distances 1.75 and $2.5 \mathrm{~mm}$ away from the center of the field of view.

corresponding excitation maximum was located in the near UVA range at $390 \mathrm{~nm}$. For that reason, use of the Opticlear ${ }^{\circledR}$ components in setups utilizing fluorescent dyes could be considered for fluorophores in which excitation and emission bands are located in the far green, red, and near infrared part of the spectrum, for example, DyLight 594 or Alexa 660. Surface quality metrics used in this article, such as RMS, $\mathrm{P}-\mathrm{V}$, and $\mathrm{Ra}$ together with aberrations coefficients indicate that tested 3-D printed lenses suffered from unwanted geometrical deformations. Wavefront deformations of the tested 3-D printed lenses were larger than wavefront deformations of a reference glass lenses. In addition, while other factors such as material uniformity may decrease the performance of these lenses to an extent, it appears that most of the performance decrease can be explained by the surface error as determined by our Zemax ${ }^{\circledR}$ simulations with measured Zernike polynomials. Based on these results, we hypothesize that the performance would significantly increase if the surface figure could be improved upon. Currently, while 3-D printed lenses cannot directly compete with off-the-shelf commercially available components, they may be an interesting alternative for those looking for asymmetric and freeform components. They can also be successfully used in illumination systems, which do not have stringent requirements for aberration corrections.

3-D printing techniques provide a great degree of flexibility. Symmetric and asymmetric objects can be manufactured in unified automated production processes. Pricing of 3-D printed parts depends on the volume of raw material rather than geometry and machining time. In addition, using 3-D printing processes, alignment features together with mounts and actuators can be built into components, allowing production of integrated modules. With 3-D printing technology in its early stage of development, we expect it to advance rapidly. We hope that 3-D printing process improvements combined with advances in material engineering will result in an improved quality of 3-D printed optics. While 3-D printed components are currently used mainly to test prototypes of opto-mechanical assemblies, we predict that this technology could be used in commercially available systems in the near future.

\section{Acknowledgments}

This work was made possible by funding from the National Institutes of Health, Grant No. R21 EB016832. 


\section{References}

1. D. Malacara and B. J. Thompson, Handbook of Optical Engineering, pp. 915-957, Marcel Dekker, Inc., New York (2001).

2. M. P. Schaub, The Design of Plastic Optical Systems, SPIE Press, Bellingham, Washington (2009).

3. H. H. Karow, Fabrication Methods for Precision Optics, John Wiley \& Sons, New York (2004).

4. S. Bäumer, Ed., Handbook of Plastic Optics, Wiley-VCH, Weinheim (2005).

5. R. E. Parks, "Overview of optical manufacturing methods," Proc. SPIE 0306, 2 (1982).

6. R. E. Wagner and R. R. Shannon, "Fabrication of aspheres using a mathematical model for material removal," Appl. Opt. 13(7), 16831689 (1974).

7. D. J. Bajuk, "Computer controlled generation of rotationally symmetric aspheric surfaces," Opt. Eng. 15(5), 401-406 (1976).

8. Satisloh, "Satisloh product catalog," http://www.satisloh.com/fileadmin/ contents/brochures/Precision_Optics/Polishing/Info_SPS-200_en.pdf (26 July 2016).

9. W. I. Kordonski and D. Golini, "Fundamentals of magnetorheological fluid utilization in high precision finishing," J. Intell. Mater. Syst. Struct. 10, 683-689 (1999)

10. D. Golini et al., "Precision optics fabrication using magnetorheological finishing," Proc. SPIE 10289, $102890 \mathrm{H}$ (1997).

11. P. R. Dumas et al., "Flexible polishing and metrology solutions for freeform optics," in Winter Topical Meeting, ASPE Free-Form Optics, Chapel Hill (2004).

12. A. Symmons, Molded Optics: Design and Manufacture, CRC Press, Boca Raton (2011)

13. A. C. Miller, "TuB1 Single point diamond turning process development," in OSA Technical Digest Series, Vol. 24, pp. 22-22, Optical Society of America (1992).

14. J. B. Arnold et al., "Machining nonconventional-shaped optics," Opt. Eng. 16(4), 164347 (1977).

15. R. Voelkel, "Wafer-scale micro-optics fabrication," Adv. Opt. Technol. 1, 135-150 (2012).

16. E. E. Sachs et al., "Three dimensional printing: rapid tooling and prototypes directly from a CAD model," J. Eng. Ind. 114(4), 481-488 (1992).

17. B. T. Wittbrodt et al., "Life-cycle economic analysis of distributed manufacturing with open-source 3-D printers," Mechatronics 23(6), 713-726 (2013).

18. 3D Systems, "3D systems materials," http://www.3dsystems.com/files/ direct-metal-brochure-0214-usen-web.pdf (27 July 2016).

19. B. Berman, "3-D printing: the new industrial revolution," Bus. Horiz. 55(2), 155-162 (2012).

20. P. Kamali et al., "The current role of three-dimensional printing in plastic surgery," Plast. Reconstr. Surg. 137, 1045-1055 (2016).
21. B. C. Gross et al., "Evaluation of 3D printing and its potential impact on biotechnology and the chemical sciences," Anal. Chem. 86(7), 3240$3253(2014)$

22. N. A. Sears et al., "A review of $3 \mathrm{D}$ printing of tissue engineering constructs," Tissue Eng. Part B 22(4), 298-310 (2016).

23. I. Gibson, D. Rosen, and B. Stucker, Additive Manufacturing Technologies: 3D Printing, Rapid Prototyping, and Direct Digital Manufacturing, Springer, New York (2014).

24. S. J. Leigh et al., "A simple, low-cost conductive composite material for 3D printing of electronic sensors," PloS One 7(11), e49365 (2012).

25. Y. Yan et al., "Additive manufacturing of magnetic components for power electronics integration," in Int. Conf. on Electronics Packaging (ICEP), IEEE (2016).

26. K. Willis et al., "Printed optics: 3D printing of embedded optical elements for interactive devices," in Proc. of the 25th annual ACM Symp. on User Interface Software and Technology, ACM (2012).

27. A. D. Squires, E. Constable, and R. A. Lewis, "3D printing of aspherical terahertz lenses and diffraction gratings," in 39th Int. Conf. on Infrared, Millimeter, and Terahertz Waves (IRMMW-THz), IEEE (2014).

28. FA. L. S. Cruz et al., "3D printed hollow core fiber with negative curvature for terahertz applications," J. Microwaves, Optoelectron. Electromagn. Appl. 14, 45-53 (2015).

29. S. F. Busch et al., "Optical properties of 3D printable plastics in the $\mathrm{THz}$ regime and their application for 3D printed $\mathrm{THz}$ optics," J. Infrared Millimeter Terahertz Waves 35(12), 993-997 (2014).

30. S. Pandey, B. Gupta, and A. Nahata, "Terahertz plasmonic waveguides created via 3D printing," Opt. Express 21(21), 24422-24430 (2013).

31. Y.-L. Sung et al., "Fabricating optical lenses by inkjet printing and heat-assisted in situ curing of polydimethylsiloxane for smartphone microscopy," J. Biomed. Opt. 20, 047005 (2015).

32. "Luxexcel material info," https://www.luxexcel.com/optics-prototyping/ material-overview (21 December 2016).

33. D. Malacara, Optical Shop Testing, 3rd ed., Wiley, New York (2007).

34. J. C. Wyant, "White light interferometry," Proc. SPIE 4737, 98 (2002).

35. Luxexcel, "Luxexcel refractive index data," http://info.luxexcel.com/ download-refractive-index-graph (21 December 2016).

John Gawedzinski received his BS degree in biomedical engineering from Boston University in 2013. He is a PhD student in bioengineering at Rice University and works in the Modern Optical Instrumentation and Bio-Imaging Laboratory led by Dr. Tomasz Tkaczyk. He research interests include the manufacturing of biomedical optical technologies used for in vivo diagnoses of diseases.

Biographies for the other authors are not available. 